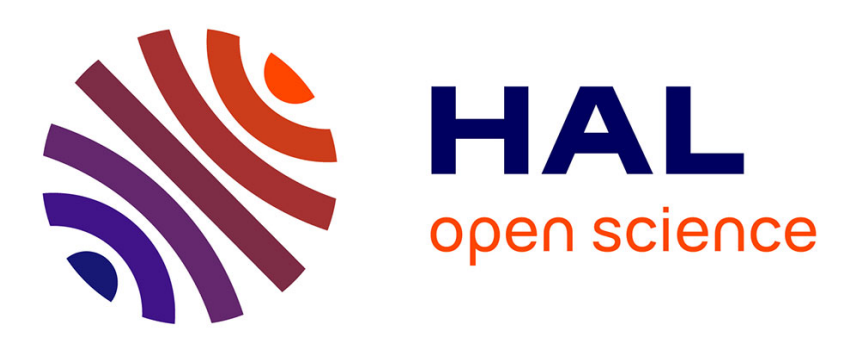

\title{
Sensorless Slowdown Detection Method for Single-Phase Induction Motors
}

Frédéric Ferreyre, René Goyet, Guy Clerc, Thierry Bouscasse

\section{To cite this version:}

Frédéric Ferreyre, René Goyet, Guy Clerc, Thierry Bouscasse. Sensorless Slowdown Detection Method for Single-Phase Induction Motors. IEEE Transactions on Energy Conversion, 2009, 24 (1), pp.60-67. 10.1109/TEC.2008.2008936 . hal-00368288

\section{HAL Id: hal-00368288 \\ https://hal.science/hal-00368288}

Submitted on 21 May 2009

HAL is a multi-disciplinary open access archive for the deposit and dissemination of scientific research documents, whether they are published or not. The documents may come from teaching and research institutions in France or abroad, or from public or private research centers.
L'archive ouverte pluridisciplinaire $\mathbf{H A L}$, est destinée au dépôt et à la diffusion de documents scientifiques de niveau recherche, publiés ou non, émanant des établissements d'enseignement et de recherche français ou étrangers, des laboratoires publics ou privés. 


\title{
Sensorless Slowdown Detection Method for Single-Phase Induction Motors
}

\author{
Frédéric Ferreyre, René Goyet, Guy Clerc, Member, IEEE, and Thierry Bouscasse
}

\begin{abstract}
The single-phase capacitor-run induction motor is generally used for fractional power in applications where the price is an important criterion. In order to save costs, one tries to estimate the speed on the basis of voltage and current measurements, and thus avoid a speed sensor. The techniques used are very varied. In the last few years, they have included numerous patents and often the use of microcontrollers. First of all, this paper shows the analytical characteristics of the induction motor with its capacitor. It explains the different possible methods to assess the speed. Then, the paper suggests a realization using one of these methods on a motor of a rolling shutter. The setup tested implements a microcontroller that cuts off the supply as soon as an abnormal reduction in speed is observed on a basis of a voltage measurement. This paper details the implementation on the industrial application.
\end{abstract}

Index Terms-Capacitor motor, induction motor, microcontroller, sensorless speed estimation, single-phase, two-phase.

\section{INTRODUCTION}

$\mathrm{C}$ APACITOR-RUN single-phase induction machines (SPIMs) are very widespread in the applications supplied by the electric network at low cost: pumps, fans, lawn mowers, home automation, etc. In this frame, it often appears to be necessary to know the speed from electric measurements. The objectives are as follows.

1) Command objectives like the starting capacitor command [1] or vector controls [2].

2) Safety objectives like operating at excessive load, jamming or blockage. For instance, on rolling shutters, the arrival of the shutter at the buffer stop results in a drop in motor speed.

The most precise method to know the speed of an induction motor consists in constructing a speed observer in transient state [3], [4]. This requires heavy calculation means as well as measurements of the two voltages and currents.

For compactness and low-cost requirements, such observers can be expensive. The command circuit must need few electric sensors and small processing means. The solution is to research into the parameters that vary with the speed.

For these low-cost applications, there are different methods to estimate the speed. All are based on the behavior of SPIM

Manuscript received November 28, 2007; revised April 11, 2008. Current version published February 19, 2009. Paper no. TEC-00464-2007.

F. Ferreyre is with the Université de Lyon, F-69622 Lyon, France, with Université Lyon 1, F-69622 Villeurbanne, France, with CNRS, UMR 5005, Laboratoire Ampère, F-69621 Villeurbanne, France, and also with Profalux, Thyez 74300, France (e-mail: frederic.ferreyre@ profalux.com).

R. Goyet and G. Clerc are with the Universite de Lyon, F-69622 Lyon, France, with Université Lyon 1, F-69622 Villeurbanne, France, and also with CNRS, UMR 5005, Laboratoire Ampère, F-69621 Villeurbanne, France (e-mail: rene.goyet@univ-lyon1.fr; guy.clerc@univ-lyon1.fr).

T. Bouscasse is with Profalux, Thyez 74300, France (e-mail: thierry. bouscasse@profalux.com).

Digital Object Identifier 10.1109/TEC.2008.2008936

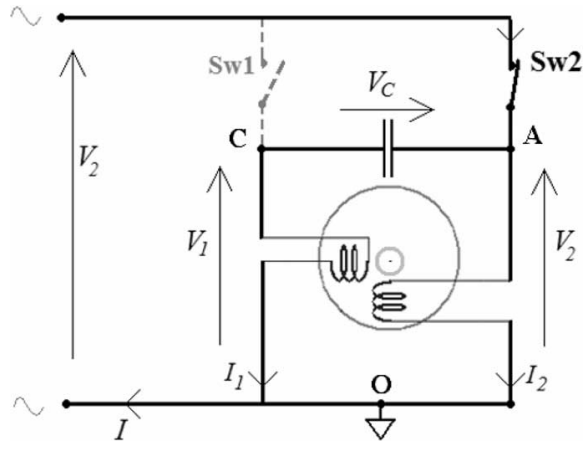

Fig. 1. Supply device of a capacitor-run single-phase induction motor.

in steady-state operation. Occasionally mentioned in patents, these methods are not yet theoretically justified and methodically compared. This paper suggests such an analysis.

SPIM operates with an unbalanced power supply, so the analysis is based on the forward and backward components. The method is classically used with SPIM, as well in previous books [5] or papers [6]-[9], as in more recent papers [10], [11].

The present study uses the terms of the impedance matrix and leads to the drawing of various characteristics representative of speed in steady state. These characteristics allow to define the criteria for choosing between the methods described in the patents [12]-[16]. A strategy is suggested to detect the slowing down of the motor with few mathematical operations. This strategy is carried out with the early detection of the arrival of a rolling shutter at the buffer stop.

\section{Modeling AND CHARACTERISTICS}

The two windings of the motor (subscript " 1 " and " 2 ") and the capacitor $C$ are often supplied from the mains with a circuit comprising two switches Sw1 and Sw2 (see Fig. 1). When the motor is symmetrical and if necessary, closing one or the other of the two switches regulates the rotational direction.

The voltages and currents of the stator are recorded with the indices 1 and 2, and their forward and backward components with the exponents + and - .

The modeling considers that the two windings are identical (two-phase symmetrical induction machine) and does not take the iron losses into consideration. In these conditions, SPIM may be represented by only two inductances, $L_{S}$ and $N$, and two resistances, $R_{S}$ and $R_{R}$. Equivalent dipoles, for forward and backward components, are those of Fig. 2(a) and (b) [5].

The primitive dipoles with three inductances are used in [6]-[11], two for leakages and one for magnetizing, and in parallel with the magnetizing inductance, a third resistance represents 


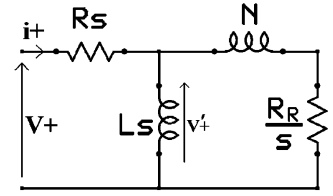

(a) (b)

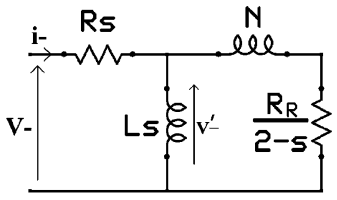

Fig. 2. Equivalent dipoles for forward. (a) Backward. (b) Components.

the iron losses. Such accuracy is not necessary in low-cost speed sensor applications.

Generally, the most relevant parameter of the induction motor is the ratio $\left(R_{R} / N \omega\right)$ between the rotor resistance $\left(R_{R}\right)$ and the total leakage impedance $(N \omega)$, with $\omega$ being the pulsation of the stator currents equal to the synchronism speed in the case of a single pair of poles.

In well-balanced steady-state operation, $\left(R_{R} / N \omega\right)$ determines the shape of the torque curve $\Gamma\left(\omega_{R}\right), \omega_{R}$ being the rotor speed. For $\left(R_{R} / N \omega\right)<1, \Gamma\left(\omega_{R}\right)$ features a maximum for $\omega_{R}<\omega$. On the contrary, when $\left(R_{R} / N \omega\right)>1, \Gamma\left(\omega_{R}\right)$ decreases uniformly when $\omega_{R}$ increases from 0 to $\omega$. The motors with a rated power of more than $1 \mathrm{~kW}$, with high efficiency, are rather of the first type, with low $R_{R}$. The motors with a rated power of less than $1 \mathrm{~kW}$ for automatic control systems are often of the second type.

In unbalanced operation with a running capacitor, the value of $\left(R_{R} / N \omega\right)$ also influences the choice of the parameters to be used to estimate $\omega_{R}$ as finely as possible. The study draws the main tendencies of the phenomenon, and justifies the different methods to estimate $\omega_{R}$, adopted in industrial realizations [13][17]. The method also depends on the structure of the command device. For example, the switches Sw1 and Sw2 that regulate the rotational direction in Fig. 1 can be placed at the bottom on the neutral side as well (point $\mathrm{O}$ ). This configuration influences the choice of the method to estimate the rotor speed $\omega_{R}$.

\section{A. Forward and Backward Components}

Calculations are turned to express the voltages and currents of the two windings, 1 and 2, according to the rotor speed $\omega_{R}$. Conversely, $\omega_{R}$ can be estimated from voltages and/or currents measurements. The system $(1,2)$ is broken up into its forward and backward symmetrical components $(+,-)$ with the complex representation [5]. The norm of the numbers represents the amplitude of the figures and not their rms value. The equations for the currents are

$$
\begin{aligned}
& \left(\begin{array}{l}
I_{1} \\
I_{2}
\end{array}\right)=\left(\begin{array}{cc}
1 & 1 \\
-j & j
\end{array}\right)\left(\begin{array}{l}
I^{+} \\
I^{-}
\end{array}\right) \\
& \left(\begin{array}{l}
I^{+} \\
I^{-}
\end{array}\right)=\frac{1}{2}\left(\begin{array}{cc}
1 & j \\
1 & -j
\end{array}\right)\left(\begin{array}{l}
I_{1} \\
I_{2}
\end{array}\right)
\end{aligned}
$$

and the same for the voltages.

The direct impedance $Z^{+}$and the inverse impedance $Z^{-}$can be expressed depending on the two $L_{S}$ and $N$ inductances and the two $R_{S}$ and $R_{R}$ resistances. The expressions are given by the equivalent dipoles of Fig. 2(a) and 2(b) [5], in which the slip
$s=1-\left(\omega_{R} / \omega\right)$ is a function of the pulsation $\omega$ of the stator currents and the rotor speed $\omega_{R}$

$$
Z^{+}=\frac{V^{+}}{I^{+}}=R_{s}+\frac{j L_{s} \omega\left(j N \omega+\left(R_{R} / s\right)\right)}{\left(R_{R} / s\right)+j\left(N+L_{s}\right) \omega}
$$

and

$$
Z^{-}=\frac{V^{-}}{I^{-}}=R_{s}+\frac{j L_{s} \omega\left[j N \omega+\left(R_{R} /(2-s)\right)\right]}{\left(R_{R} /(2-s)\right)+j\left(N+L_{s}\right) \omega} .
$$

The motor impedance matrix is

$$
\left(\begin{array}{l}
V_{1} \\
V_{2}
\end{array}\right)=\left(\begin{array}{cc}
A & B \\
-B & A
\end{array}\right)\left(\begin{array}{c}
I_{1} \\
I_{2}
\end{array}\right)
$$

with $A=\left(Z^{+}+Z^{-}\right) / 2$ and $B=j\left[\left(Z^{+}-Z^{-}\right) / 2\right]$.

If the phase 2 is supplied by the mains, in parallel with the phase 1 connected in series with the capacitor $C$, this gives

$$
V_{2}=V_{1}+Z_{L} I_{1}
$$

where $Z_{L}=1 / j C \omega$.

The preceding equations result in some "specific ratios" that facilitate characterizing the operational points

$$
\begin{aligned}
& \frac{I_{1}}{I_{2}}=\frac{A-B}{A+B+Z_{L}} \\
& \frac{V_{1}}{V_{2}}=\frac{A^{2}+B^{2}+B \times Z_{L}}{A^{2}+B^{2}+A \times Z_{L}} \\
& Z_{1}=\frac{V_{1}}{I_{1}}=\frac{A^{2}+B^{2}+B \times Z_{L}}{A-B} \\
& Z_{2}=\frac{V_{2}}{I_{2}}=\frac{A^{2}+B^{2}+A \times Z_{L}}{A+B+Z_{L}} \\
& \frac{I}{V_{2}}=\frac{1}{Z}=\frac{2 A+Z_{L}}{A^{2}+B^{2}+A \times Z_{L}}
\end{aligned}
$$

where $I=I_{1}+I_{2}$ is the total current absorbed by the motor.

The mean power $P_{R}$ absorbed at the rotor includes Joule effect losses and mechanical power. $P_{R}$ is a function of the currents $I_{1}, I_{2}, I^{+}, I^{-}$, and the voltages $V^{\prime}$ at the terminal of $L_{S}$ inductances (see Fig. 2).

1) There are two $V^{\prime}$ voltages for the real motor:

$$
V_{1}^{\prime}=V_{1}-R_{S} I_{1} \quad V_{2}^{\prime}=V_{2}-R_{S} I_{2} .
$$

2) There are two $V^{\prime}$ voltages for the equivalent dipole of Fig. 2:

$$
\begin{aligned}
V^{++} & =V^{+}-R_{S} I^{+} \quad V^{\prime-}=V^{-}-R_{S} I^{-} \\
P_{R} & =\frac{1}{2} \operatorname{Re}\left[V_{1}^{\prime}\left(I_{1}^{*}\right)+V_{2}^{\prime}\left(I_{2}^{*}\right)\right] \\
& =\operatorname{Re}\left[V^{\prime+}\left(I^{+*}\right)+V^{\prime-}\left(I^{-*}\right)\right] .
\end{aligned}
$$

Re indicates "real part" and * indicates "conjugate."

The torque is the difference between its forward and backward components

$T=\frac{\operatorname{Re}\left[V^{\prime+}\left(I^{+}\right)^{*}\right]}{\omega}+\frac{\operatorname{Re}\left[V^{\prime-}\left(I^{-}\right)^{*}\right]}{-\omega}$ 
$T=\frac{1}{\omega}\left(\left|I^{+}\right|^{2} \operatorname{Re}\left(Z^{+}-R_{s}\right)-\left|I^{-}\right|^{2} \operatorname{Re}\left(Z^{-}-R_{s}\right)\right)$

$T=\frac{1}{\omega}\left(\left|\frac{V^{+}}{Z^{+}}\right|^{2} \operatorname{Re}\left(Z^{+}-R_{s}\right)-\left|\frac{V^{-}}{Z^{-}}\right|^{2} \operatorname{Re}\left(Z^{-}-R_{s}\right)\right)$.

In other words, by introducing the rms value $V_{2 \mathrm{rms}}$ of the ac power voltage $V_{2}(t)$

$$
\begin{aligned}
T=\frac{V_{2 \mathrm{rms}}^{2}}{2 \omega}\left(\left|\frac{\left(V_{1} / V_{2}\right)+j}{Z^{+}}\right|^{2} \operatorname{Re}\left(Z^{+}-R_{S}\right)\right. \\
\left.-\left|\frac{\left(V_{1} / V_{2}\right)-j}{Z^{-}}\right|^{2} \operatorname{Re}\left(Z^{-}-R_{S}\right)\right) .
\end{aligned}
$$

This expression for $T$ slightly differs from [9] and [10], but it can easily be shown that, with no stator leakage inductance and no difference between the windings 1 and 2, the expressions are similar.

Some remarks about the power transfer and the mathematical transformations are as follows.

1) In (1) and (2)

$$
P=\left(\begin{array}{cc}
1 & 1 \\
-j & j
\end{array}\right) \quad P^{-1}=\frac{1}{2}\left(\begin{array}{cc}
1 & j \\
1 & -j
\end{array}\right)
$$

only separate the forward and backward components on condition that $I_{1}$ and $I_{2}$ are expressed with the complex representation.

2) The transformations $P$ and $P^{*}$ are such as

$$
P_{T} P^{*}=\left(\begin{array}{ll}
2 & 0 \\
0 & 2
\end{array}\right) \text {. }
$$

This gives $V_{1}\left(I_{1}^{*}\right)+V_{2}\left(I_{2}^{*}\right)=2\left(V^{+}\left(I^{+*}\right)+V^{-}\left(I^{-*}\right)\right)$ and a factor $1 / 2$ in (12). Another transformation

$$
P^{\prime}=\frac{1}{\sqrt{2}}\left(\begin{array}{cc}
1 & 1 \\
-j & j
\end{array}\right)
$$

may be used. It is such as

$$
P^{\prime-1}=\frac{1}{\sqrt{2}}\left(\begin{array}{cc}
1 & j \\
1 & -j
\end{array}\right) \quad P_{T}^{\prime} P^{\prime *}=\left(\begin{array}{ll}
1 & 0 \\
0 & 1
\end{array}\right)
$$

and then the factors 2 and 1/2 disappear in complex power expressions.

\section{B. Voltage and Current Curves for a Rolling Shutter Motor}

Although the rolling shutter SPIM is geometrically and magnetically balanced, its identification from four parameters is not obvious. First of all, a fifth parameter is theoretically necessary to represent the iron losses. Then, the parameters, specifically the stator resistance, depend on the temperature. The identification is nevertheless possible from experimental current and voltage measurements. It is made in order to maximize the conformity between measures and calculated data, from zero speed to synchronism at every temperature. This gives the results of Table I.

A dc measurement of the stator resistance gives a value $250 \Omega$ quite less than $R_{S}$ in Table I. This underlines the sig-
TABLE I

$R_{S}, L_{S}, N$, AND $R_{R}$ AS A FUnCtION OF THE TEMPERATURE $\theta$

\begin{tabular}{|c|c|c|c|c|}
\hline $\boldsymbol{\theta}\left({ }^{\circ} \mathbf{C}\right)$ & $\mathbf{R}_{\mathbf{S}}(\boldsymbol{\Omega})$ & $\mathbf{L}_{\mathbf{s}}(\mathbf{H})$ & $\mathbf{N}(\mathbf{H})$ & $\mathbf{R}_{\mathbf{R}}(\boldsymbol{\Omega})$ \\
\hline 25 & 275 & 1,534 & 0,072 & 475 \\
\hline 50 & 305 & 1,625 & 0,030 & 459 \\
\hline 90 & 337 & 1,689 & 0,080 & 503 \\
\hline
\end{tabular}

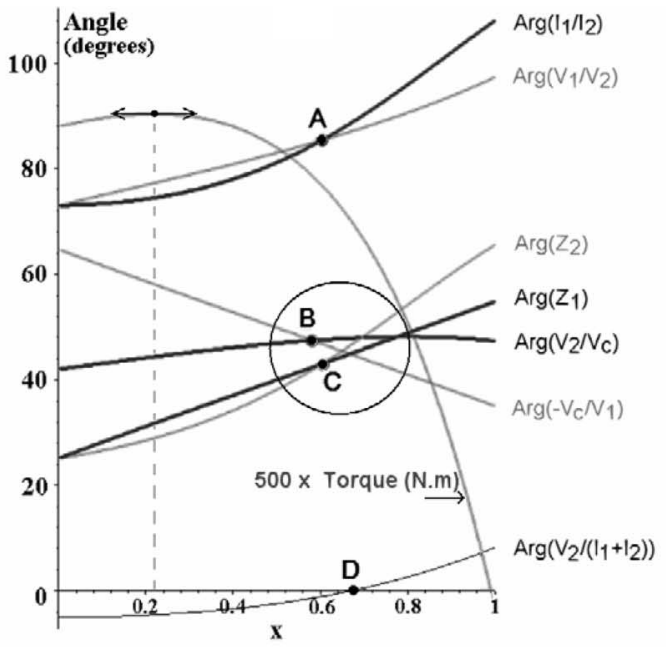

(a)

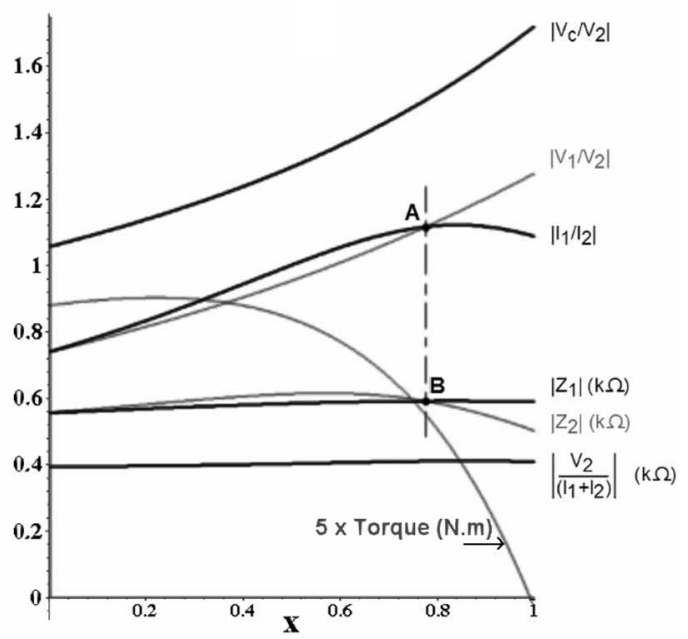

(b)

Fig. 3. (a) Torque and arguments of differing complex numbers characteristics, ratios of voltages, currents or impedances, depending on the reduced speed $x=$ $\omega_{R} / \omega=1-s$. (b) Amplitude of differing complex numbers, ratios of voltages, currents or impedances, depending on the reduced speed $x=\omega_{R} / \omega=1-s$.

nificance of the iron losses and shows the limit of an identification from four parameters. We have, however, adopted this four-parameter model as it is practically analytical and makes it easier to represent in a sufficiently accurate manner the various operational regimes.

The analytical model is used to calculate the curves of Fig. 3. The four parameters are those of the first line of Table I, at $\theta=25^{\circ} \mathrm{C}$, and the capacitor is $C=4 \mu \mathrm{F}$.

Fig. 3(a) and 3(b) confirms the variations, depending on the operating point, of several "specific complex numbers" (7)(11), shown in the preceding paragraph. Fig. 3(a) gives their arguments and Fig. 3(b) their amplitude. The relative speed 


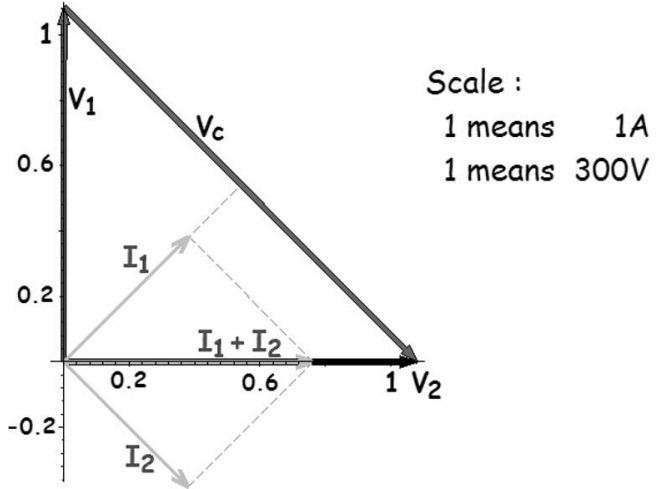

Fig. 4. Ideal conditions without backward component.

$x=\omega_{R} / \omega=1-s$ is shown on the $X$-axis. These analytical results turn out to be very close to the measurements. Indeed, even if the model is not exactly realistic, the parameters are identified for their concordance.

The torque $T(x)$ shows a maximum for $x=0.2$ [see Fig 3(a)]. This comes from the unbalanced regime. On the contrary, with a balanced power supply, the ratio $\left(R_{R} / N \omega\right)=21$ is fairly high to eliminate any maximum in the torque curve $T(x)$ (as mentioned in Section II).

With $C=4 \mu \mathrm{F}$, no speed meets the ideal condition that eliminates the backward component and leads to a global power factor equal to 1 . This ideal regime is designated by

$\alpha_{1}=\arg \left(Z_{1}\right)=\arg \left(Z_{2}\right)=\arg \left(\frac{V_{2}}{V_{C}}\right)=\arg \left(\frac{-V_{C}}{V_{1}}\right)=45^{\circ}$

$\alpha_{2}=\arg \left(\frac{I_{1}}{I_{2}}\right)=\arg \left(\frac{V_{1}}{V_{2}}\right)=90^{\circ}$

$\alpha_{3}=\arg \left(\frac{V_{2}}{I_{1}+I_{2}}\right)=0^{\circ} \quad k=\left|\frac{I_{1}}{I_{2}}\right|=\left|\frac{V_{1}}{V_{2}}\right|=\left|\frac{Z_{1}}{Z_{2}}\right|=1$

and corresponds to the Fresnel diagram in Fig. 4 without backward component.

However, another capacitance $C_{1}$ may give this ideal regime for a specific speed $x_{1}$ and a specific torque value. The values of $C_{1}$ and $x_{1}$ are $C_{1}=3.72 \mu \mathrm{F}$ and $x_{1}=0.65$. Given this operating condition, Fig. 3 presents a quite different shape.

In Fig. 3(a), the group of points around B and C, inside the circle, joins together. This unique point, the point $\mathrm{A}$, and the point $\mathrm{D}$ are aligned on the same vertical with the characteristics $\alpha_{1}=45^{\circ}, \alpha_{2}=90^{\circ}$, and $\alpha_{3}=0^{\circ}$.

In Fig. 3(b), the ordinate of A would be $k=1$.

The nominal speed of the SPIM of this paper is very close to these ideal operating conditions.

The curves Fig. 3 facilitate the estimate of the variations of each value with the speed $x$. These variations are more often than not monotonic, and each value can be used to assess the relative speed $x$. Research into the most accurate measure is made easier by plotting the Fresnel diagrams. This is done in the next paragraph.

\section{Fresnel Diagrams for Different Motors}

In the complex plane, the three voltages $V_{2}=\mathrm{OA}, V_{1}=$ $\mathrm{OC}$, and $V_{C}=\mathrm{CA}$ form a triangle $\mathrm{OAC}$ that is distorted when the relative speed $x$ varies. Fig. 5 gives the configuration of the voltage triangles obtained for three motors, at two different speeds: $x=0$ (notation ') and $x=1$ (notation without $^{\prime}$ ). Fig. 5 also gives the three complex currents: $I_{1}, I_{2}$, and $I=I_{1}+I_{2}$.

The Fresnel diagram Fig. 5(b) corresponds to the rolling shutter SPIM of this paper with $\theta=25^{\circ} \mathrm{C}, C=4 \mu \mathrm{F}, L_{S 0}=$ $1.535 \mathrm{H}, N_{0}=0.072 \mathrm{H}, R_{S 0}=275 \Omega$, and $R_{R 0}=475 \Omega$.

The two other motors of Fig. 5(a) and (c) are close to this one. As explained in Table II, one or two parameters are changed.

The motor of Fig. 5(a) has a lower inductance $L_{S}=1.195 \mathrm{H}$ instead of $L_{S 0}=1.535 \mathrm{H}$.

The motor of Fig. 5(c) has lower stator and rotor resistances: $R_{S}=41 \Omega$ instead of $R_{S 0}=275 \Omega$. The assumption is realistic as, for rolling shutter tubular motors, the size is reduced and $R_{S}$ is usually high. Besides, it facilitates the running on locked rotor at nominal voltage.

The motors in Fig. 5(a) and (c) have a specific behavior. The Table II highlights this behavior comparing it to the case of Fig. 5(b).

For the motor in Fig. 5(a), the points $\mathrm{C}$, for $x=1$, and $\mathrm{C}^{\prime}$, for $x=0$, are on the same straight line $\mathrm{CA}=\mathrm{C}^{\prime} \mathrm{A}$. The two angles

$$
(\mathrm{CAO})=\arg \left(\frac{V_{2}}{V_{C}}\right) \quad\left(\mathrm{C}^{\prime} \mathrm{AO}\right)=\arg \left(\frac{V_{2}}{V_{C}^{\prime}}\right)
$$

are equal $\left(38^{\circ}\right)$, and their measurement does not make it possible to estimate the relative speed $x$. In fact, during the passage from $x=1$ to $x=0$, the trajectory of point $\mathrm{C}$ deviates a little from the straight line passing through $\mathrm{A}$, but the measurement of $x$ by $(\mathrm{CAO})$ remains not very sensitive.

In the same way for Fig. 5(c), the two angles for $x=1$ and $x=0,(\mathrm{AOC})=\arg \left(V_{1} / V_{2}\right)$ and $\left(\mathrm{AOC}^{\prime}\right)$, are equal $\left(98^{\circ}\right)$, and the measurement of the relative speed $x$ by (AOC) is not possible. The motor is "low" resistant. Instead, in this case, the angle (AOC) can be useful for determining approximately when the torque is maximal, especially in the management of capacitors in capacitor-start induction motors [1].

The situation where the two angles (OCA), for $x=1$, and $\left(\mathrm{OC}^{\prime} \mathrm{A}\right)$, for $x=0$, are equal also exists. It is found, for example, in the case of $L_{S}=1.3 L_{S 0}, N=4.17 N_{0}, R_{S}=0.098 R_{S 0}$, and $R_{R}=0.098 R_{R 0}$. One finds OCA $=\mathrm{OC}^{\prime} \mathrm{A}=36^{\circ}$. The Fresnel diagram is not shown in Fig. 5. In this case, the measurement of $x$ by (OCA) is not possible.

In the case of the rolling shutter SPIM of this paper [see Fig. 5(b)], the angle (CAO) changes from $47^{\circ}$ for $x=1$ to $42^{\circ}$ for $x=0$. It does not, therefore, vary very much, and its measurement is not advisable for an estimation of $x$.

The differences of reaction between motors explain the large number of patents lodged on the subject. The discussion is not easy in as much as few details are given on the motors in question. In spite of everything, we can suggest these simple explanations, for example, in the following two patents: Tecumseh makes pumps and cold section refrigerating motors. They suggest measuring the relative $x$ speed on the basis of the angle (CAO). The explanation is that $R_{S}$ is low enough for the measurement to be sensitive [12]. 

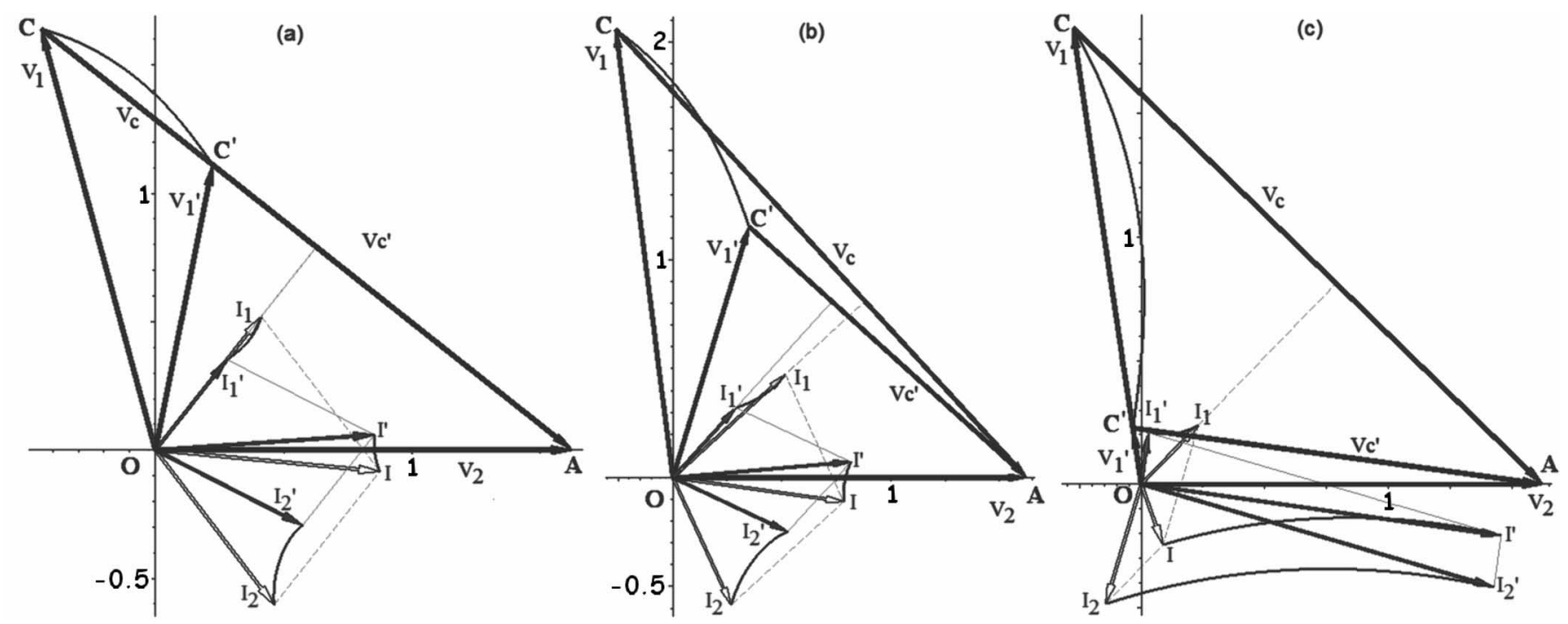

Fig. 5. Fresnel diagrams of voltages and currents with their trajectories for $x \in[0 ; 1]$. Scale: for currents "1" means 1 A, and for voltages "1" means $200 \mathrm{~V}$. The vectors at the synchronism speed $(x=1)$ are noted without asterisk $\left(^{\prime}\right)$ and those of null speed $(x=0)$ are noted with an asterisk $\left({ }^{\prime}\right)$.

TABLE II

RELEVANCE OF THE MEASUREMENTS DEPENDING ON THE TYPE OF MOTOR

\begin{tabular}{|c|c|c|c|c|c|c|c|c|c|c|}
\hline \multirow[b]{2}{*}{ Fig. } & \multirow[b]{2}{*}{$\operatorname{Rs}(\Omega)$} & \multirow[b]{2}{*}{ Ls (H) } & \multirow[b]{2}{*}{$\mathbf{N}(\mathrm{H})$} & \multirow[b]{2}{*}{$R^{\prime}(\Omega)$} & \multirow[b]{2}{*}{$C(\mu F)$} & \multicolumn{2}{|c|}{$A \hat{O} C=\arg \left(V_{1} / V_{2}\right)$} & \multicolumn{2}{|c|}{$C A \hat{O}=\arg \left(-V_{c} / V_{2}\right)$} & \multirow[b]{2}{*}{ Relevant measurements } \\
\hline & & & & & & $x=1$ & $x=0$ & $x=1$ & $x=0$ & \\
\hline $5 . a$ & 275 & 1.195 & 0.072 & 475 & 4 & 105 & 79 & 38 & 38 & AÔC, |V1|, |Vc| \\
\hline $5 . b$ & 275 & 1.535 & 0.072 & 475 & 4 & 97 & 73 & 47 & 42 & AÔC, $|\mathrm{V} 1|,|\mathrm{Vc}|$ \\
\hline 5.c & 41 & 1.535 & 0.072 & 71 & 4 & 98 & 98 & 44 & 8 & $|\mathrm{~V} 1|,|\mathrm{Vc}|, \mathrm{CÂO}$ \\
\hline
\end{tabular}

Bubendorf makes "high $R_{S}$ " rolling shutter motors, which avoids the measurement of $x$ by (CAO) and suggests (AOC) [14].

A third patent, Plumer Company [13], gives all the possible solutions for the measurement of relative speed $x$ with the phase differences (arguments) of the complex numbers (7)-(11), but it does not confirm how to choose one rather than the other. The company insists rather on the signal processing techniques to estimate the variations of the value measured (sliding derivative).

European patents in [15] and [16] suggest to use the phase between voltages and currents in order to prevent the blockage of SPIM.

In conclusion, with SPIM, there are many relevant measurements of phase or amplitude, of voltages or currents, to detect the speed. Nevertheless, the speed estimation by the voltages is generally preferable for reasons of cost.

\section{SLOWING-DOWn CRITERIA}

\section{A. Method}

The data processing of measurements in rolling shutter motion is complicated due to many reasons.

1) The motor parameters change with the temperature.

2) The running of the slats is very irregular.

3) There are many different kinds of motors and shutters.
Measures have to be compared with thresholds and filtered in order to eliminate doubtful acquisition and noise. There are certainly many suitable signal processing methods for such elimination. The one presented in this paper is not so simple but has the merit of being safe for a wide range of shutters and operating conditions.

The previous study has shown that for rolling shutter SPIM, the speed could be determined on notably the basis of $\arg \left(V_{1} / V_{2}\right)$ or $\left|V_{1} / V_{2}\right|$, values that are adapted for these values decrease if the speed decreases [see Fig 5(b)]. Therefore, they are used to detect an excessive decrease in speed and cut off the supply of the motor. In this application, the decrease represents the arrival of the rolling shutter on the buffer stop.

The same signal processing, applied to the two values, leads each time to a stoppage criterion. The stoppage is effective if the two criteria are checked simultaneously. In the case of the rolling shutters, it has appeared that this "AND" function reduced the risk of spurious stoppage.

The criterion used is explained later by showing indifferently by " $y$ " one or other of the two measurements. These measurements are renewed every $\Delta t=10 \mathrm{~ms}$, the half-period of the $50 \mathrm{~Hz}$ supply. At the instant $t_{i}$, the measurement $y_{i}$ can be analyzed in several ways. For example, it is possible:

1) to calculate the absolute difference $\left(y_{i}-y_{0}\right)$, between $y_{i}$ and a fixed low value $y_{0}$, very much lower than the nominal operational conditions. This technique is not definite as there is a risk of the reaction being too late; 


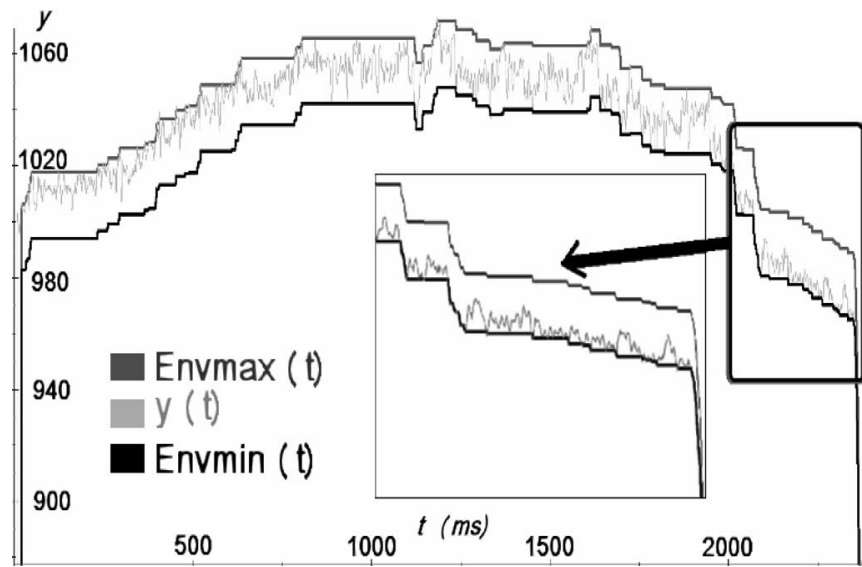

Fig. 6. Input signal and output envelopes for the noise reduction stage--(Env max) and (Env $\min$ ) surround $y ; y$ : arbitrary vertical scale.

2) to compare the relative difference between two consecutive measurements and a threshold $S$; in other words, to calculate

$$
E(i)=\left[\left(y_{i}-y_{i-1}\right)-S\right] .
$$

Stop the motor when $E(i)>0$. This technique is not suitable either as, whatever the value of $S$, even very low, there is always an insignificant slope

$$
p_{i}=\frac{y_{i}-y_{i-1}}{\Delta t}
$$

repeated at each $t_{i}$, so as to always have $E(i)<0$. In this way, it is possible to attain 0 speed without cutout. Even more, with a threshold $S$ too small, there is a risk that the system might stop on the noise component.

3) To calculate several values

$$
E(i, j)=\left[\left(y_{i}-y_{i-j}\right)-S\right]
$$

with $j$ varying from 1 to $N$. Stop the motor at the first $j=J$ if $E(i, J)>0$. The threshold $S$ and the number of times $N$ where $E(i, j)$ is tested at each value of $i$ are predefined. $N$ and $S$ depend on $p_{i}$. With the rolling shutters, the situation is complicated by the irregularities in speed caused by the unwinding of the slats. The choices of $N$ and $S$ are delicate; they can even require to consider functions $N(i)$ and $S(i, j)$.

The third method has been chosen with fixed $N=18$ and variable $S$ with $j$, which is referred to as $S(j)$.

\section{B. Implementation}

The measurements, recorded every $10 \mathrm{~ms}$, are very noisy. Fig. 6 gives an example of a typical measure of $\arg \left(V_{1} / V_{2}\right)$ or $\left|V_{1} / V_{2}\right|$; both are similar, and the scale is arbitrary. The measure, named $y$, illustrates the signal processing carried out. The latter comprises three stages 1)-3).

1) Reduction in Noise: The noise reduction has been first realized with filtering techniques, like sliding average over a great number of values of $y$. It appears that the method involves a long, unacceptable delay, in comparison with the desired reac- tion time of the system. Then, another method has been adopted. It consists of replacing the noisy $y$ signal by two other signals without noise surrounding y, called EnvMax and EnvMin that are the lower and the upper bound of $y$.

For that, at each $y_{i}$, three values are calculated

$$
\begin{aligned}
\text { sliding average: } & S y_{i}=\frac{y_{i}+y_{i-1}}{2} \\
\text { difference: } & D y_{i}=\frac{\left|y_{i}-y_{i-1}\right|}{2} \\
\text { thickness: } & E y_{i}=\max \left(D y_{i}\right) .
\end{aligned}
$$

These three values are a function of the evolution of $y$ and the background noise. They are used to calculate the envelopes, EnvMax and EnvMin. The calculation is made on the basis of the following logic.

1) If $S y_{i}>E n v M a x_{i}$, then

$$
\left\{\operatorname{EnvMax}_{i+1}=S y_{i} \text { and } \operatorname{EnvMin}_{i+1}=S y_{i}-E y_{i}\right\} \text {. }
$$

2) If $S y_{i}<E n v M i n_{i}$, then

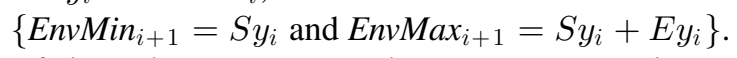

Fig. 6 shows how EnvMin $i$ and EnvMax ${ }_{i}$ surround $y$.

The following study is carried out on the basis of EnvMin ${ }_{i}$. During a continuous decrease in $y, E n v M i n_{i}$ follows $S y_{i}$, which itself is very near to $y_{i}$. Once initialized in the first measurements, the thickness $E y_{i}$ hardly changes at all in practice in such a way that the system operates as a hysteresis follower with two outputs, one being the limit inferior and the other the limit superior of $y$. When the instantaneous variation of $y$ exceeds $E y_{i}$, EnvMin updates this variation with the smallest possible delay $(10 \mathrm{~ms})$.

2) Buffering and Detection: The reduction in speed is studied from $N$ successive measurements of $y$ stored in the form of

$$
\alpha_{i} \operatorname{EnvMin}_{i}
$$

with $\alpha_{i}=1$ if $y$ decreases, i.e., if $\left(S y_{i}<E n v M i n_{i-1}\right)$, and $\alpha_{i}=$ 0 in the opposite case.

This arrangement means taking into account only the decreasing measurements of $y_{i}$. This has turned out to be beneficial in the case of rolling shutters to reduce calculation time and eliminate the irregularities of speed.

After each $\operatorname{EnvMin}_{i}$ storage, $N$ comparisons of $\alpha E n v M i n$ to $N$ different thresholds $S(j)$ are made.

The motor is stopped if the following condition is verified:

$$
\left(\alpha_{i-j} \operatorname{EnvMin}_{i-j}-\operatorname{EnvMin}_{i}\right)>S_{j} .
$$

The comparison is not made if $\left(\alpha_{i-j}\right.$ EnvMin $\left._{i-j}=0\right)$.

Fig. 7 indicates the process. At the top, the storage memory is presented with only 13 of the 18 cells.

Later, the 13 corresponding thresholds are given that represent the maximum differences of speed permitted. The differences $\left(\alpha_{i-j} \operatorname{EnvMin}_{i-j}-\operatorname{EnvMin}_{i}\right)=\left(\alpha_{i-j} \operatorname{EnvMin}_{i-j}-1039\right)$ between the two are indicated each time they are calculated. The first time the difference equals 12 , the threshold 13 , and move on to the second calculation. At the fourth calculation, the difference 24 is higher than the threshold 20, the process is stopped, 


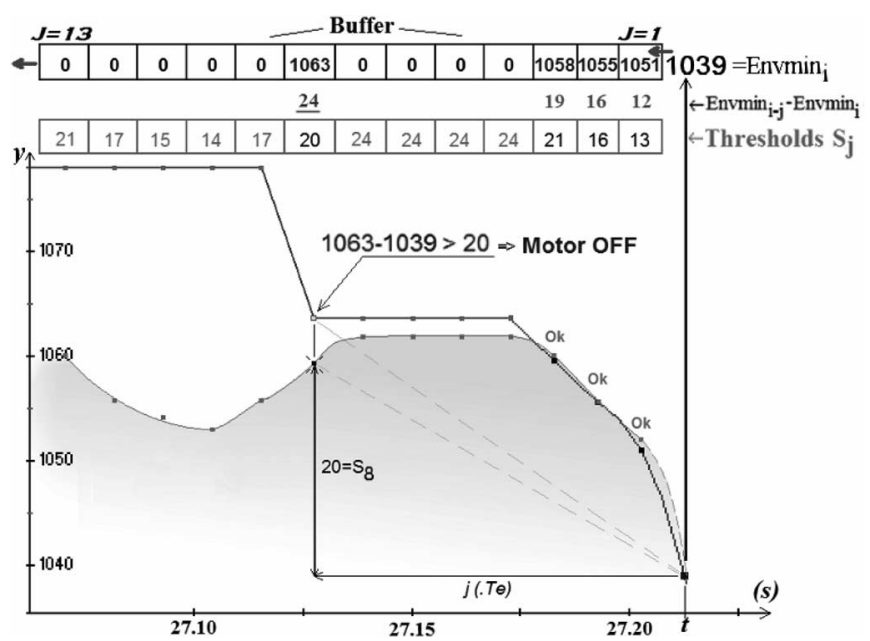

Fig. 7. Multiple thresholds comparison; $y$ : arbitrary vertical scale.

and the motor turned off. The lower part of the figure shows the preceding calculations and the profile of the thresholds adopted.

3) Controller of Thresholds: When the speed includes a lowfrequency component associated with a load irregularity, it is advisable to adapt the series of thresholds $S(j)$. The procedure can be made automatically due to a threshold controller that analyzes the speed variations, and decides consequently, to load a set of precalculated $N$ thresholds rather than another.

In the case of rolling shutters, we adopted $M=4$ sets of $N=18$ thresholds. Every $360 \mathrm{~ms}$, the series of $N \operatorname{EnvMin}_{i}$ is analyzed and the set of $N$ thresholds is changed if necessary.

The efficiency of the slowing-down detection algorithm strongly depends on the threshold values. These are determined on the basis of a statistical study of about a thousand measurements, performed on many rolling shutters in operation, with different motors of the same family, at different temperatures. The $N \times M$ thresholds are calculated with a reciprocal algorithm so that they encompass all the configurations of tested rolling shutters, motors, and temperatures, without adjustment or reprogramming of the command board.

\section{EXPERIMENTAL VALIDATION ON A ROLLING SHUTTER}

\section{A. Equipment}

The previous study is implemented in practice to detect the arrival of a rolling shutter on the buffer stop, without the sensor. The experimental bench is made up of an SPIM motor supplied by the ac power with an inversion device for the rotational direction (see Fig. 1). The drive board shown in Fig. 8 is made around an 8-bit microcontroller Microchip PIC16F876. It decodes and interprets the user orders sent by the radio. Two circuits convert and filter the voltages $V_{1}$ and $V_{2}$ to 0-5 $\mathrm{V}$ compatible levels. They are connected to the analog-to-digital (ADC) inputs and the two programmable capture inputs of the microcontroller. This gives access to the phase difference between $V_{1}$ and $V_{2}$ and their amplitudes.

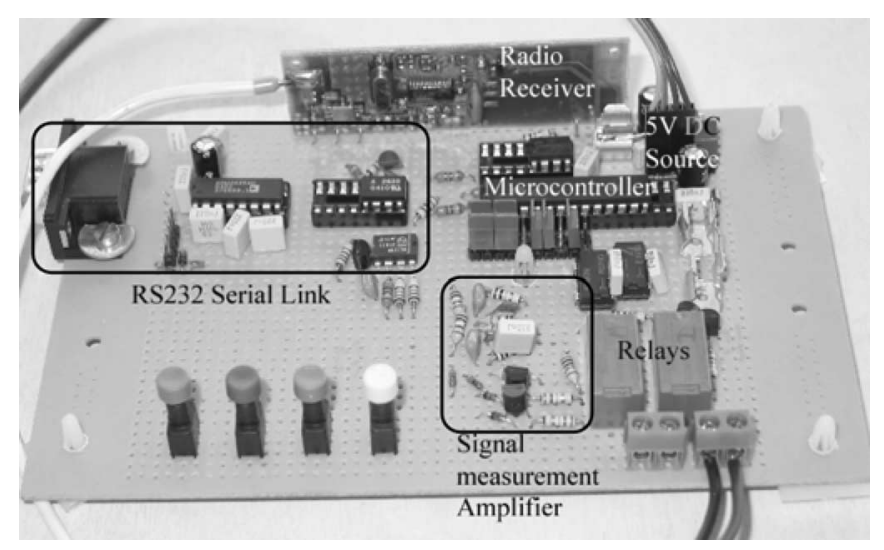

Fig. 8. Prototype of the command board.

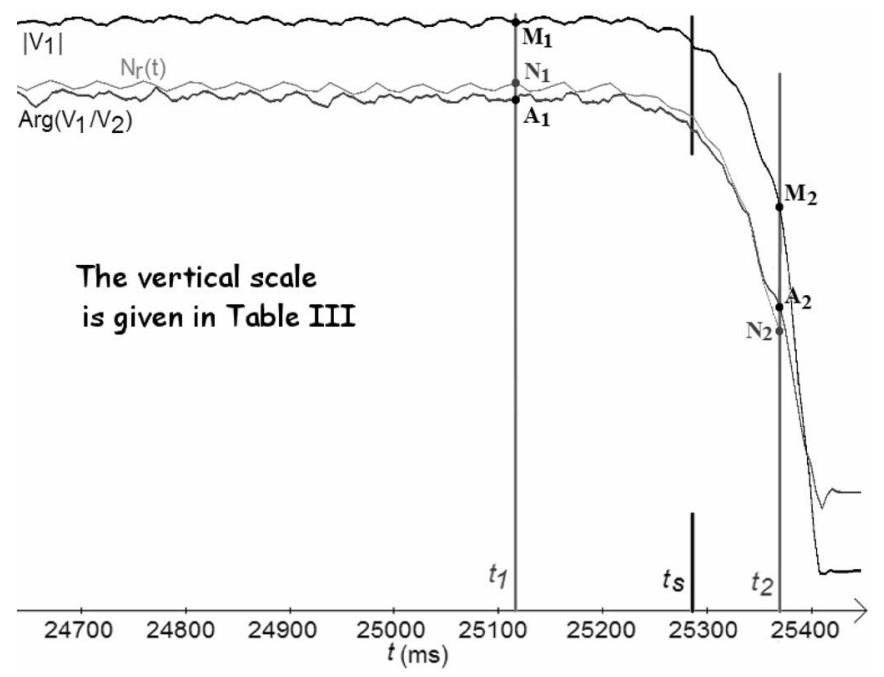

Fig. 9. Speed and voltage of the auxiliary phase $V_{1}(t)$, for blocking the shutter when lowering without cutout.

\section{B. Results}

In order to validate the slowdown detection method (SDM), thousands of tests have been achieved on various configurations of loads, motors, and temperatures. Each time, the stoppage successfully happens, approximately $40-90 \mathrm{~ms}$ after the beginning of the slowdown. When the shutter reaches the buffer stop, the reaction torque on the motor strongly increases. Due to the celerity of the SDM, this torque is kept lower than its nominal value (about $0.1 \mathrm{~N} \cdot \mathrm{m}$ ). Without SDM, it would reach the maximum $(0.2 \mathrm{~N} \cdot \mathrm{m})$. Experimentally, this result is satisfactory. The shutter is clamped on the buffer stop without mechanical distortion or audible grating.

A test without SDM is given Fig. 9 and Table III. It allows to study the whole slowing-down process and measure the efficiency of an eventual SDM. The speed, the phase difference $\arg \left(V_{1} / V_{2}\right)$, and the amplitude of $V_{1}$ are recorded at the time of a forced stoppage. The rotor speed is measured by an incremental encoder giving one pulse per revolution. The slowing down starts a little after $t_{1}=25.1 \mathrm{~s}$. The last speed measured $N_{2}$ is at $t_{2}=25.4 \mathrm{~s}-$ beyond this, accuracy is no longer ensured. 
TABLE III

CharaCteristic POINTS OF FIG. 9

\begin{tabular}{|c|c|c|c|}
\hline Abscissa $t$, (ms) & $t_{1}=25120$ & $t_{s}=25290$ & $t_{2}=25370$ \\
\hline Speed $N_{r}(t),(r p m)$ & $N_{1}=2678$ & 2560 & $\mathrm{~N}_{2}=1808$ \\
\hline$\left|V_{1}\right|(t),(V)$ & $M_{1}=380$ & 374 & $M_{2}=333$ \\
\hline $\operatorname{Arg}\left(V_{1} / V_{2}\right)(t),\left(^{\circ}\right)$ & $A_{1}=85.5$ & 84.1 & $A_{2}=75.8$ \\
\hline
\end{tabular}

Fig. 9 and Table III clearly illustrate that the phase difference and the norm of $V_{1}$ have the same characteristics as that of the speed, with a measurement delay of 10-30 ms. The theoretical results of Section II are confirmed. On the recording, we can see that SDM, with the most sensitive of the four thresholds series, would stop the motor in $t_{S}=25290 \mathrm{~ms}$, e.g., $60 \mathrm{~ms}$ after the beginning of the slowing down.

When the temperature increases, the curves $\arg \left(V_{1} / V_{2}\right)$ and $\left|V_{1} / V_{2}\right|$ are slightly shifted down, but as the algorithm makes comparisons between values, this turns out to have no effect on the criteria.

\section{CONCLUSION}

This paper shows a system for assessing the speed and detecting the slowing down applied to the single-phase induction motor with a running capacitor. The appraisal of the speed reposes on the measurement of the voltages. The disposition is researched in numerous industrial scenarios to save on the speed sensor. The study starts by an analytic modeling of the evolution in the voltages and currents with the speed. It then appears that the speed can be estimated on the basis of voltages and currents of the stator or the capacitor. The value used is determined from the parameters of the motor.

The implementation is not simple, since the values measured are disturbed by noise. This paper suggests an original and systematic method for reducing the impact of noise. It shows an algorithm that responds straight away to an excessive slowing down while remaining resistant to the electrical parasites and the irregularities of speed imposed by the load. Generally speaking, the thresholds and the background history of the $N$ dimensions are adjusted according to the load profile, which makes the algorithm suitably adaptable to any type of application. The method is applied in this case to a rolling shutter motor, the objective being an end-of-travel stop. The objective has been reached with an assembly using a microcontroller, and the measurement results are shown with related comments.

\section{REFERENCES}

[1] J. P. Borrelli and R. Burkhart, "New phase-sensitive technology for capacitor start motor simplifies application," IEEE Trans. Ind. Appl., vol. 34, no. 2, pp. 253-257, Mar./Apr. 1998.

[2] M. B. de Rossiter Corrêa, C. B. Jacobina, E. R. C. da Silva, and A. M. N. Lima, "Vector control strategies for single-phase induction motors drive systems," IEEE Trans. Ind. Electron., vol. 51, no. 5, pp. 10731080, Oct. 2004.

[3] A. Payman and S. Vaez-Sadeh, "DSP based speed estimation of single phase induction motors," in Proc. 2004 IEEE 35th Annu. Power Electron. Spec. Conf. (PESC 2004), vol. 2, pp. 1335-1340.

[4] S. Vaez-Sadeh and S. H. Reicy, "Sensorless vector control of single-phase induction motor drives," in Proc. 8th IEEE Int. Conf. Electr. Mach. Syst. (ICEMS 2005), vol. 3, pp. 1838-1842.
[5] P. Vas, Electrical Machines and Drives: A Space Vector Theory Approach. Oxford, U.K.: Clarendon, 1992, pp. 502-511.

[6] H. Huang, E. F. Fuchs, and J. C. White, "Optimal placement of the run capacitor in single phase induction motor design," IEEE Trans. Energy Convers., vol. 3, no. 3, pp. 647-652, Sep. 1988.

[7] E. F. Fuchs, A. J. Vandenput, J. Höll, and J. C. White, "Design and analysis of capacitor-start, capacitor-run single-phase induction motors," IEEE Trans. Energy Convers., vol. 5, no. 2, pp. 327-336, Jun. 1990.

[8] L. Xu, "Dynamic model of an integrated-cycle controlled single phase induction machine," IEEE Trans. Energy Convers., vol. 7, no. 4, pp. 761767, Dec. 1992.

[9] D.-H. Jang and J.-S. Won, "Voltage, frequency, and phase-difference angle control of PWM inverters-fed two-phase induction motors," IEEE Trans. Power Electron., vol. 9, no. 4, pp. 377-383, Jul. 1994.

[10] C. Mademlis, I. Kioskeridis, and T. Theodoulidis, "Optimisation of singlephase motors, Part 1: Maximum energy efficiency control," IEEE Trans. Energy Convers., vol. 20, no. 1, pp. 187-195, Mar. 2005.

[11] C. Mademlis, I. Kioskeridis, and T. Theodoulidis, "Optimisation of singlephase motors, Part 2: Maximum energy efficiency control," IEEE Trans. Energy Convers., vol. 20, no. 1, pp. 196-203, Mar. 2005.

[12] D. Nehring, "Microprocessor based motor control system with phase difference detection," U.S. Patent 5808 441, Sep. 15, 1998.

[13] D. Nassr, P. Raude, and L. Plumer, "Control device for stopping the operation of a single-phase asynchronous motor with a capacitor," U.S Patent 5760 556, Jun. 2, 1998.

[14] P. Raude and A. Mansouri, "Stop control device for single-phase asynchronous motor with capacitor," French Patent 2785 469, Mar. 5, 2000.

[15] M. Jacob and S. Dutertre, "Stop control device for single-phase asynchronous motor with capacitor," French Patent 2838 886, Oct. 24, 2003.

[16] T. Stempniakowski, "Control device for an electric motor powered by ac," French Patent 2813 453, Mar. 1, 2002.

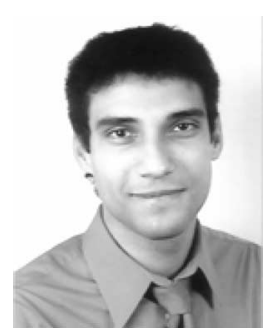

Frédéric Ferreyre was born in 1982. He received the Engineering degree from the Ecole Catholique d'Arts et Métiers de Lyon (ECAM) Engineering School, Lyon, France, in 2003. He is currently working toward the Ph.D. degree at the University Claude Bernard Lyon 1, Villeurbanne, France.

$\mathrm{He}$ is also currently with Profalux, where he is engaged in driving induction motors.

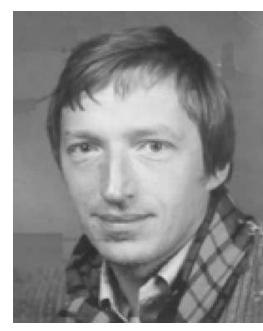

René Goyet was born in France, in 1946. He received the Engineering degree from Ecole Speciale des Travaux Publics de Paris (ESTP), Paris, France, in 1969, and the "These d'Etat" (long Ph.D.) from the University of Paris 6, Paris, in 1981.

He was an Assistant Professor at the University of Paris 6 for 30 years. He is currently with the University Claude Bernard Lyon 1, Villeurbanne, France. He has also been a teacher of electronics, electrotechnics, and automatics. His current research interests include driving motors and magnetic modeling for electrical engineering. He has authored or coauthored numerous technical publications.

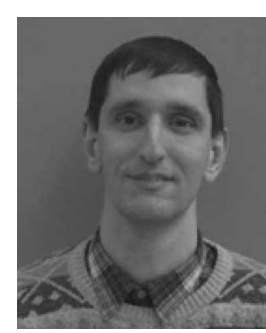

Guy Clerc (M'00) was born in Libourne, France, on November 30, 1960. He received the Engineering and Ph.D. degrees in electrical engineering from the Ecole Centrale de Lyon, Lyon, France, in 1984 and 1989 , respectively.

He is currently a Full Professor, and teaches electrical engineering at the University Claude Bernard Lyon 1 (UCBL), Villeurbanne, France, where he is engaged in research on control and diagnosis of induction machines at Laboratoire Ampere.

Thierry Bouscasse was born in 1951. He is the Chairman of Profalux, Thyez, France. 